\title{
The space as a natural laboratory of Electrotechnics
}

\author{
P. K. Marhavilas*
}

Dep. of Electrical Eng., Technological Educational Institute of Kavala, Greece Space Research Lab., Democritus University of Thrace, Xanthi, Greece.

Received 29 October 2007; Accepted 14 January 2008

\begin{abstract}
Electricity is a general term for a variety of phenomena resulting from the presence and flow of charge, and "Electrotechnics" is the study or the science of practical and industrial applications of electricity. On the other side, the space is mainly characterized by the interaction of energetic charged particles with electric and magnetic fields, which justifies the claim «the space composes a natural laboratory of electrotechnics». This paper reviews present understanding of the dynamics of the solar-terrestrial environment, and its impacts on the human activity, and explain processes, which establish the space as a natural laboratory of electrotechnics.
\end{abstract}

Keywords: Electrotechnics ; Solar activity ; Solar Wind ; Sunspots ; Prominences ; Solar Flares ; Coronal Mass Ejections (CMEs) ; Geomagnetic storms and substorms; Space Weather; Aurora; Space Weather

\section{Introduction}

Electricity is a general term [1] for a variety of phenomena resulting from the presence and flow of charge. This includes many well-known physical phenomena such as electromagnetic fields, electric currents and lightning, and is used in industrial applications such as electronics and electric power. These concepts are better identified by more precise terms as follow:

- Electric charge: a connection conserved property of some subatomic particles, which determines their electromagnetic interactions. Electrically charged matter is influenced by, and produces, electromagnetic fields, while the movement (or flow) of electrically charged particles composes the electric current.

- Electric potential: the capability of an electric field to do work.

- Electrical energy: the energy made available by the flow of electric charge through an electrical conductor.

- Electromagnetism: One of the four fundamental interactions/forces of nature (the others are the gravitation, the weak interaction, and the strong interaction).

Moreover, Electrotechnics is the study or the science of practical and industrial applications of electricity.

On the other side, the space is principally characterized by the interaction of energetic charged particles with

*E-mail address: marhavil@ee.duth.gr ISSN: 1791-2377 @ 2008 Kavala Institute of Technology. All rights reserved. electric and magnetic fields [2], which justifies successfully the claim "the space constitutes a natural laboratory of electrotechnics". In the vicinity of Earth, most of these charged particles derive their energy ultimately from the sun or from the interaction of the solar "wind" with the earth's magnetosphere. These interactions are complex, because the magnetic and electric fields that determine the motion of the particles are affected in turn by the motion of these particles.

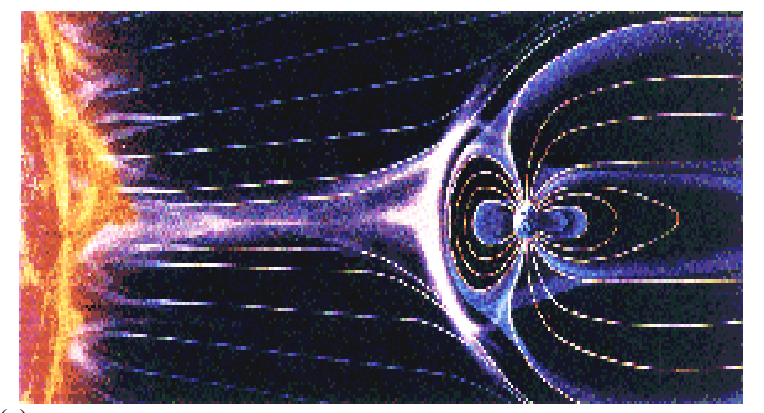

(a)

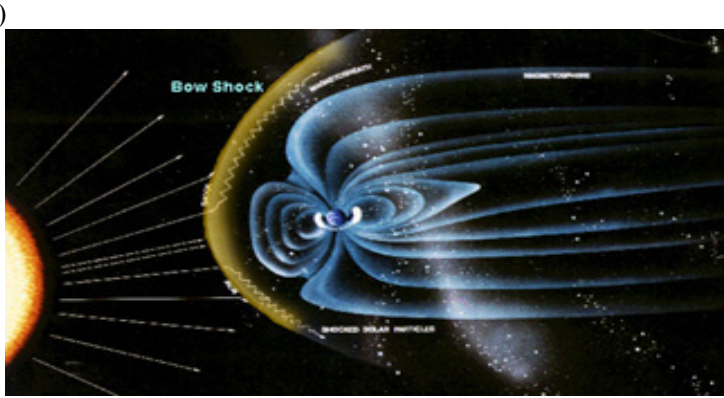

(b)

Fig 1. An artist illustration of the Sun-Earth connection. (see [7], [8], [9], [10]) 
The life on Earth is intimately connected to the way the earth responds to the Sun's variations. We live in the extended atmosphere of an active star, as we can realize from the artist illustration of the Sun-Earth connection of Figure 1 [3]. While sunlight enables and sustains life, the Sun's variability produces streams of high-energy particles and radiation that can harm life or alter its evolution. Under the magnetosphere (a protective shield of a magnetic field) and atmosphere, Earth is an island in the Universe. (http://sec.gsfc.nasa.gov/sec_science.htm).

As the sphere of the human environment and exploration continues to expand space, the understanding effects of our active Sun and "space weather" on power and communications, astronaut safety, satellite operations, and climate change, become day after day more important. We need to understand and be able to predict the effects of solar activity on Earth and society.

This paper reviews present understanding of the dynamics of the solar-terrestrial environment, and its impacts on the human activity, and explain processes, which constitute the space as a natural laboratory of electrotechnics.

\section{The stormy sun}

Nowadays, we know that the Sun is a class G star [4], has a mass of $1.98 \times 10^{30} \mathrm{kgr}(\sim 333,000$ times larger than the Earth's mass), a radius of $\sim 695,000 \mathrm{~km}$ (while Earth's radius is $6378 \mathrm{~km}$ ), and composed of ionized hydrogen and helium gas located $\sim 150$ million $\mathrm{km}$ from the Earth. It is an enormous gaseous sphere, which rotates faster around the equator (27 days) than it does at the poles ( $\sim 35$ days). Its surface temperature is about $5,770^{\circ}$ Kelvin and its interior temperature is about 16 million degrees Kelvin. Its total radiated energy is equivalent to 100 billion tons of TNT exploding per second. This power is important to us because it supports the life on Earth. It causes seasonal changes, ocean current flows, atmospheric circulation, and photosynthesis for plant life.

The Sun contains several major sections, the core along with the radiative and the convective layers, and constitutes a huge thermonuclear reactor. The process by which the Sun gives off energy is the fusion i.e. the conversion of hydrogen into helium by nuclear reactions, which release energy. Even though the nuclear burning occurs in the core, the heat and light generated from this process take about 10 million years to reach the Sun's surface. Once the photons depart the core, they must travel through the radiative layer to the convective zone where the temperatures go from 8 million to $7,000^{\circ}$ Kelvin. After reaching the Sun's surface also known as the photosphere, the photons travel through the chromosphere and it eventually reach the corona. The last three regions with their different physical properties constitute the solar atmosphere. Most of the solar radiation comes from the photosphere, which emits a continuous spectrum with superimposed dark absorption lines. The photosphere is the visible surface of the Sun. This is what we see in a clear day from the ground. Its temperature is $\sim 5,800{ }^{\circ} \mathrm{K}$. The chromosphere lies above the photosphere up to a height of $\sim 1,500 \mathrm{~km}$ with a temperature of $\sim 10,000$ to $\sim 500,000{ }^{\circ} \mathrm{K}$. When the Sun is observed through filters of different wavelengths, pictures can be obtained of the Sun's structure at a variety of levels. In the beginning of an eclipse we can see light that has emitted from the photosphere and is then scattered towards us at the chromospheric levels as well as the intrinsic chromospheric emission. The corona is the upper layer of the solar atmosphere. In this layer the temperature rises to more than $\sim 1,000,000 \mathrm{~K}$.

\section{The Solar Wind}

Near its surface, the Sun is like a pot of boiling water, with bubbles of hot, electrified gas - actually electrons and protons in the forth state of matter known as "plasma" - circulating up from the interior, rising to the surface, and bursting out into space. The steady stream of ionized plasma, which continuously escapes from the solar corona and pervades the whole interplanetary space [5], [31], is known as the solar wind. This gas, composed of electrons and protons with approximately $10 \%$ helium ions, also induces geomagnetic activity by variations of its pressure and magnetic field [6]. At the orbit of Earth, this wind has a density of about 10 particles per $c c$, a temperature of about $1 \mathrm{keV}$ (equivalent to about $10,000,000 \mathrm{~K}$ ) and an average speed of about 500 $\mathrm{km} / \mathrm{sec}$. The solar wind gas also has magnetic fields from the sun imbedded within it. At Earth's orbit, these fields have a strength of $\sim 10 \mathrm{nT}$ (about 3,000 times less than the Earth's magnetic field at its surface).

Blowing at $\sim 400$ to $\sim 2,500 \mathrm{~km} / \mathrm{sec}$, the solar wind (see Figure 2) carries a million tons of matter into space at rate of $1.6 \times 10^{12} \mathrm{gr} / \mathrm{sec}[7]$ and energy at a rate of $1.8 \times 10^{27} \mathrm{erg} / \mathrm{sec}$, that is negligible in the overall mass and energy balance of the sun. It's not the mass or speed, however, that makes the solar wind potent. In fact, the solar wind would not even ruffle the hair on the head because there are too few particles in the breeze (our air is millions of times denser than the solar wind). Instead, it is the energy stored in the plasma and the magnetic fields associated with that plasma that allow the wind to shape and impact Earth's protective magnetic shield in space (the magnetosphere). Though less than $1 \%$ of the solar wind penetrates the magnetosphere, that's enough to generate millions of amps of electric current in atmosphere and to cause occasional storms in the space around Earth [8].

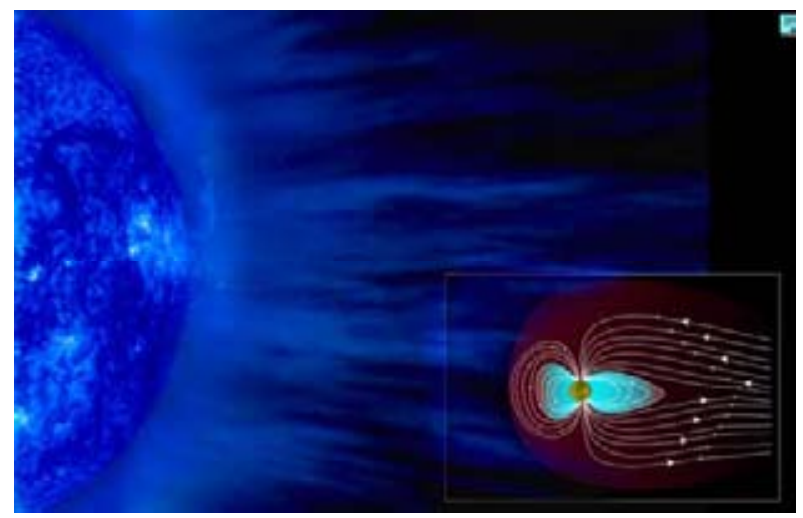

Fig 2. The solar wind streaming away from the Sun. [7]

The Sun-Earth system is driven by the 11-year solar cycle. This means that every 11 year the Sun reaches a peak period of activity called "solar maximum", followed a few years later by a period of quiet called the "solar minimum". During solar maximum there are many sunspots, eruptive prominences, solar flares, and coronal mass ejections (CMEs), all of which can affect communications and other technology here on Earth. The last maximum occurred in 2000 during Cycle 23. 


\section{Sunspots}

One way of tracking solar activity is by observing the number of sunspots. During solar maximum there are hundreds of sunspots and during solar minimum only a dozen can be found. In the photosphere the sunspots are characterized by the most intense concentrations of magnetic flux that have been formed during the emergence of flux in one day or so. They are relatively cool areas that appear as dark patches like freckles on the solar surface formed when magnetic field lines just below the Sun's surface are twisted and poke through the solar surface (see Figure 3). They appear dark because they are not as hot or bright as the area surrounding them $\left(4,000^{\circ}\right.$ Kelvin vs. $6,000^{\circ}$ Kelvin). Sunspots can last from a few hours to several months, and a large sunspot can grow several times the size of Earth. Though the Chinese recorded some observations as early as 28 B.C., scientists have been observing and recording sunspots since the $17^{\text {th }}$ Century. (Galileo, who first performed scientific observations in the early 1613 , concluded that the Sun did indeed have spots.) The scientists care about sunspots because they are visible signs of the turmoil inside the Sun that lead to space weather effects on Earth [9].

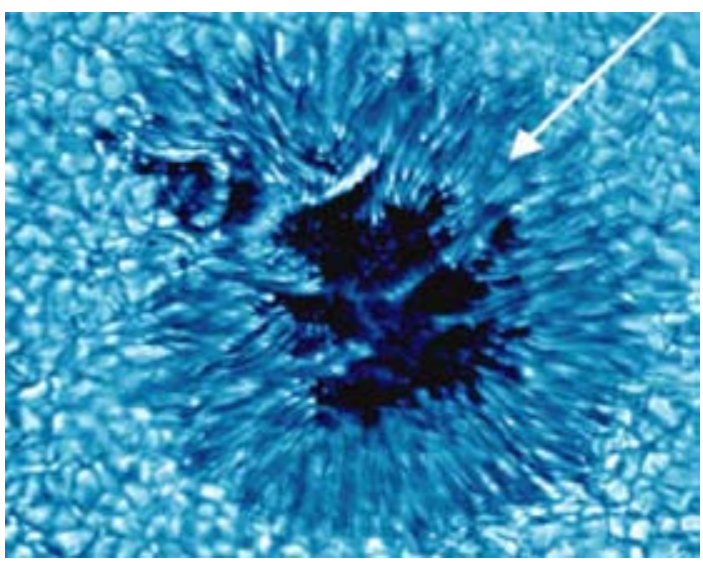

(a)

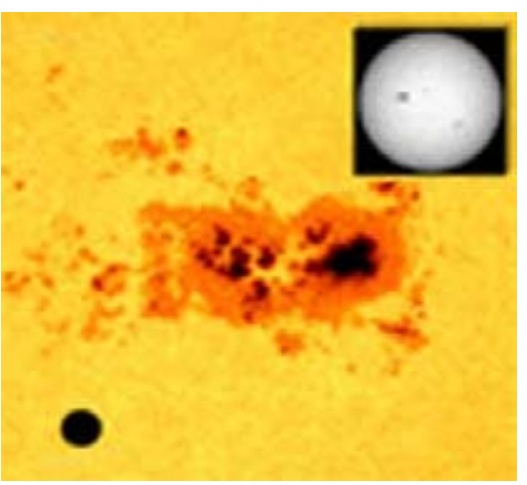

(b)

Fig 3. a) A large sunspot (http://sohowww.nasacom.nasa.gov; http:// sohowww.estec.esa.nl), b) The biggest sunspot group in 10 years (2001/03/27, 12:48 UT), exceeded 13 times the size of Earth. (see [7]. [8], [9], [10]).

However sunspots, which are surrounded by areas with enhanced brightness, are not the only element of solar activity. The Sun emits more energy when it is active. Increased solar activity also means stronger and more frequent eruptive prominences, and solar flares (a dramatic release of energy equivalent to a million hundred-megaton nuclear explosions).

\section{Prominences}

Prominences are the most impressive objects on the Sun because they are located in the corona and possess temperatures a hundred times lower and densities a hundred or a thousand times greater than the coronal values. They are structures consisting of cool plasma supported by magnetic fields (see Figure 4) and bright when seen at the Sun's edge. However, when seen against the bright solar disk they are dark and are called filaments. If they have broken away from the sun, they are called eruptive prominences.
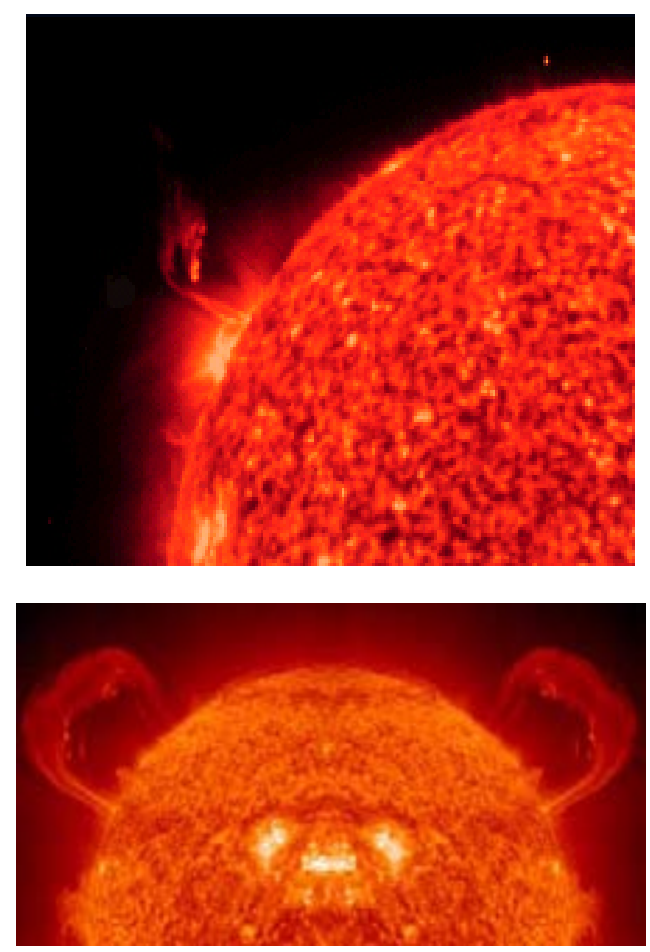

Fig 4. An image of a large eruptive prominence emerging from the solar surface that was taken by the Extreme Ultraviolet Imaging Telescope onboard NASA's Solar and Heliospheric Observatory (SOHO) spacecraft, in an extreme ultraviolet wavelength of ionised helium heated to about 60,000 to $80,000 \mathrm{~K}$.

(http://sohowww.nasacom.nasa.gov, http://sohowww.estec.esa.nl)

\section{Solar Flares}

Solar Flares are huge explosions in the Sun's atmosphere. They appear to our instruments as bright flashes in visible light, often followed by a burst of high-energy protons and radiation. Moreover their characteristics can include bursts of radiowaves, EUV and X-rays. A large solar flare can release a thousand million megatones of energy (more precisely $10^{28}$ to $10^{34} \mathrm{ergs}$ ) in a single explosion. The released energy is transformed into: 1) thermal energy (localized heating leading to an increased brightness of e.g. the Ha and X-ray emission), 2) particle kinetic energy leading to the acceleration of electrons to energies of $10 \mathrm{keV}$ to $1 \mathrm{GeV}$ and ions to energies from a few $\mathrm{MeV} /$ nuc to $\mathrm{GeV} /$ nuc, 3) mechanical energy leading to several kinds of plasma ejecta. Solar flares sometimes occur together with other signatures of solar activity e.g. prominence eruptions, CMEs and inter- 
planetary shock waves. However the exact relationship between these phenomena is not yet completely understood. In fact the solar flares are one of the main challenges of space weather prediction. Figure 5 shows the unusually large flare, which took place on 14/7/2000 [10]. This event was so remarkable that it has become known as the "Bastille Day" event.

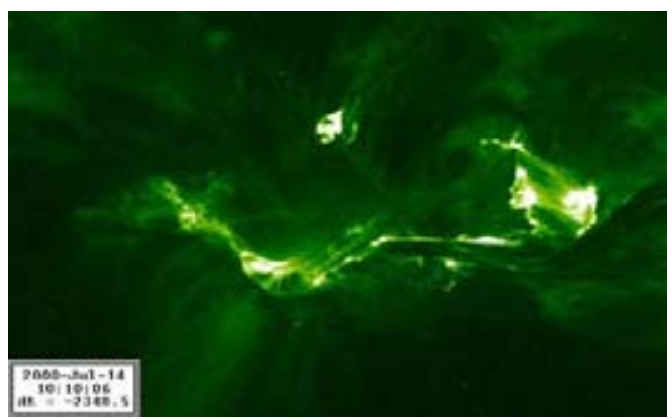

Fig 5. The unusually large flare, which took place on 14 July 2000 . This event was so remarkable that it has become known as the "Bastille Day" event. (see [7], [8], [9], [10])

\section{Coronal Mass Ejections (CMEs)}

One of the most important solar events from the Earth's perspective is the coronal mass ejection, the solar equivalent of a hurricane [9]. A CME is the eruption of a huge bubble of plasma from the Sun's outer atmosphere. It can occur with or without solar flares, and can threaten Earth's atmosphere. Once it escapes the Sun's gravity, a CME speeds at velocities approaching $400 \mathrm{~km} / \mathrm{sec}(\sim 1,000,000$ miles $/ \mathrm{hr})$ up to $2,000 \mathrm{~km} / \mathrm{sec}(\sim 5,000,000 \mathrm{miles} / \mathrm{hr})$. A typical CME can carry more than 10 billion tons of plasma into the solar system. Just hours after blowing into space, a CME cloud can grow to dimensions exceeding those of the Sun itself, often as wide as 20 million $\mathrm{km}$ across. As it ploughs into the solar wind, a CME can create a shock wave that accelerates particles to dangerously high energies and speeds. Behind that shock wave, the CME cloud flies through the solar system bombarding planets, asteroids, and other objects with radiation and plasma. If a CME erupts on the side of the Sun facing Earth, and if our orbit intersects the path of that cloud, the results can be spectacular and sometimes hazardous. Figure 6 shows an artist illustration of a CME, and also a large CME erupting from the Sun. The CME is seen as a bright cloud expanding towards the right hand side of the image taken using the LASCO coronograph onboard SOHO. In this case an EUV image of the Sun has been superimposed onto the LASCO image to show the size and location of active regions on that day.

\section{Geomagnetic Field, Geomagnetic storms and substorms}

The region near-Earth space, where the dynamics is governed by the internal geomagnetic field, is called magnetosphere. The solar wind flow past the Earth distorts the dipole field to compress it on the dayside and elongate it to a long geomagnetic tail on the nightside. The geomagnetic tail plays a key role in magnetospheric dynamics; for example, it acts as an energy reservoir for the dynamic processes [11]. The magnetosphere (Figure 7) comprises distinct regions, which all have their characteristic plasma properties: The tail lobes at high latitudes are regions of low plasma density and energy, whereas the plasma sheet is characterized by denser and hotter $\sim \mathrm{keV}$ plasma.

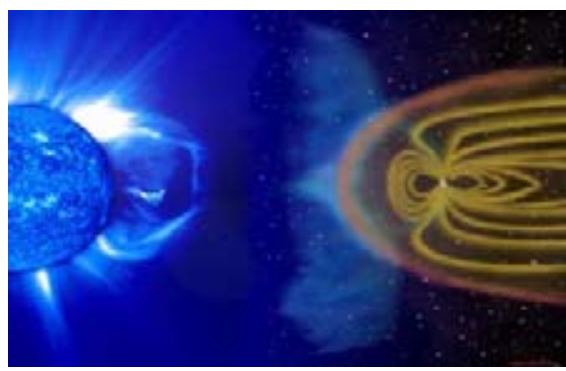

(a)

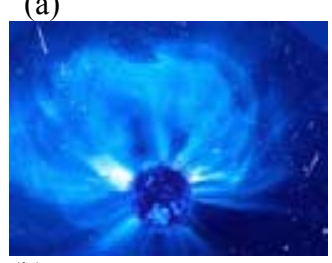

(b)

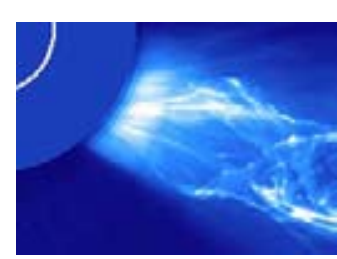

(c)
Fig 6. a) An artist illustration of a coronal mass ejection. b), c) Large CMEs erupting from the Sun. A CME is seen as a bright cloud expanding towards the right hand side of the image taken using the LASCO coronograph onboard SOHO.

(http://sohowww.nasacom.nasa.gov, http://sohowww.estec.esa.nl)

The geomagnetic field influences particle motions in the Earth's orbit, and it also deflects some particles from interplanetary space. The geomagnetic field would have a dipole shape except for the solar wind that distorts the geomagnetic field into a parabolic shape. The magnetic field traps charged particles, mostly electrons and protons, into the Van Allen radiation belts. The magnetic field is strongest at low altitudes. The dipole field geometry results in a region, known as the South Atlantic Anomaly (SAA), where the radiation belts reach their lowest altitude. The South Atlantic Anomaly (SAA) is a region of particularly intense proton flux. The figure $7(a, b)$ shows an overview of the Earth's magnetosphere [12].

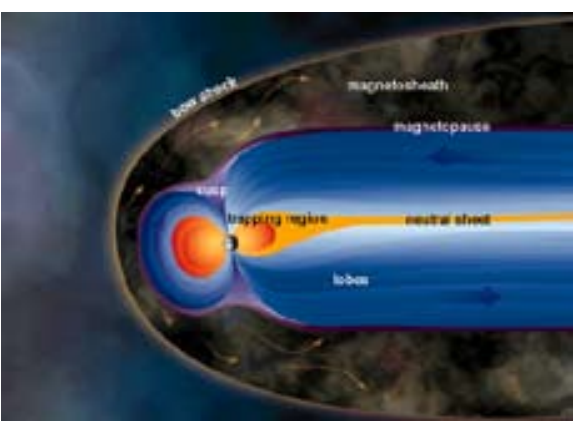

(a)

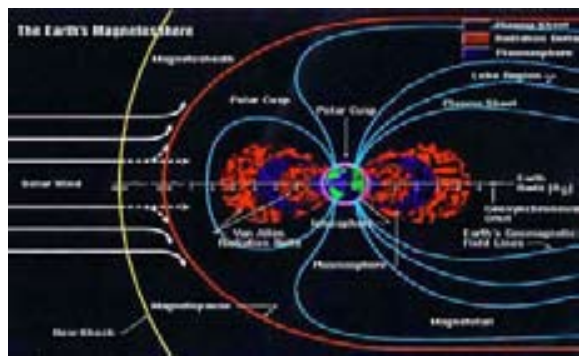

(b)

Fig 7. (a), (b) [12] Schematic of the Earth's proximate space environment, the magnetosphere. 
The most hazardous region for technological systems is the inner magnetosphere, where trapped populations of highenergy (from hundreds of $\mathrm{keV}$ to multi-MeV) electrons and ions reside in the ring current and in the Van Allen radiation belts.

The dynamic response of the magnetosphere to varying solar wind and interplanetary magnetic field conditions is the magnetospheric substorm [13]. Energy input from the solar wind is largely controlled by the interplanetary magnetic field orientation: During periods of southward interplanetary field, the energy input is enhanced and the energy extracted from the solar wind is stored in the magnetosphere in the form of magnetic field energy in the magnetotail. This is the substorm growth phase. After typically 30-60 min, the magnetotail undergoes a change of state from stable to unstable, and the stored energy is dissipated via a highly dynamic process. This substorm expansion phase involves an injection of energetic (tens to hundreds of $\mathrm{keV}$ ) electrons and ions to the vicinity of the geostationary orbit, strong electric currents in the auroral regions, and rapid fluctuations and configurational changes of the magnetospheric magnetic field. All these phenomena are potential space weather effects. The substorm process ends when the energy dissipation ceases and the magnetosphere recovers its initial state after about two to four hours from the beginning of the event (interesting reviews: [11], [14], [30]).

Geomagnetic storms are large disturbances in the nearEarth environment caused by coherent solar wind and interplanetary field structures that originate from solar disturbances such as CMEs [15]. Storms are associated with major disturbances in the geomagnetic field and strong enhancement of the fluxes of energetic (tens to hundreds of $\mathrm{keV}$ ) ions and high-energy (up to several $\mathrm{MeV}$ ) electrons in the outer Van-Allen radiation belt [16].

\section{Impacts on human life and activity}

\section{Space Weather}

As A. Frank has written [17], humanity is maturing into a space-faring race. The response of the space environment, particularly around the Earth, to the stormy Sun, is known as Space Weather, which is a hot topic today, because of the increasing awareness that many modern technological systems are potentially vulnerable to the effects from solar storms. On the other side, the danger of some aspects of space weather has slowly been recognized, and is studied by the scientists in order to help protect space and ground systems (technical and biological) from space environment hazards. Space weather disturbances are generally caused by transient events in the solar atmosphere. There are two different types of events, which trigger disturbances in the Earth's environment [18] : a) solar flares and b) CMEs. However, not all solar flares result in geomagnetic storms, and even more significantly, not all geomagnetic storms can be associated with solar flares. CMEs are some of the most dramatic space weather effects. The emission from the two types of disturbances can be divided into two classes: a) particle radiation and $b$ ) electromagnetic radiation, which will have different effects on the Earth's environment.

Particle Radiation: A continuous flow of charged particles (protons and electrons), the solar wind, is streaming out from the Sun. Moreover, several types of solar events can cause particles with high velocities to be superimposed on this background solar wind. CMEs carry billion tons of mat- ter at high speeds, considerably greater than the normal solar wind velocities. The cloud of charged particles (which also bring with them parts of the solar magnetic field) interacts with the Earth's magnetic field when it reaches the Earth's orbit. This results in a disturbance of the Earth's magnetic field, and the auroral particle precipitation into the atmosphere increases. The aurora (as discussed below) is a dynamic and delicate visual manifestation of solar-induced geomagnetic storms.

Electromagnetic Radiation: The energetic radiation bursts from flares, travel at the speed of light well ahead of any particles or coronal material associated with the flares, arrive at Earth just 8 minutes after leaving the flare site. Moreover, unlike the electrons and ions of the solar wind and the solar energetic particle populations, the passage of electromagnetic waves is not affected by the presence of Earth's magnetic field. The direct response of the upper atmosphere to a burst of solar flare ultraviolet and x-ray emissions is a temporary increase in ionisation (as well as temperature) in the sunlit hemisphere, lasting from minutes to hours and called a sudden ionospheric disturbance. This can cause disruption of short-wave radio communication at $\mathrm{HF}$ frequencies (3-30 MHz), which is still extensively used by the military and for overseas broadcasting.

\section{Historical Notes}

The earliest evidence for the impact of solar-terrestrial phenomena on technical systems appeared in the first half of the $19^{\text {th }}$ century [19]. Beginning with the invention of telegraphy in 1841, during solar storms, "earth currents" induced by the changing terrestrial magnetic field, were so powerful that telegraphers didn't need a battery to send their messages down the line. Any relationship of the sun to the appearances of the "spontaneous" currents that were measured on the telegraph wires was not clarified until the occurrence of the large white light solar flare of late August 1859 (Carrington, 1863; the first such flare ever recorded by astronomers [20]). Within a day following this flare, large geomagnetic disturbances and wide-spread aurora were observed over the Earth, at low geomagnetic latitudes in Hawaii and Rome. The advancement in communications provided by radio stimulated a significant need to better understand the medium that was critical in bending the radio waves around the curvature of Earth, the ionosphere. The same ionosphere currents that could produce "spontaneous" Earth currents could also affect the reception and the fidelity of the transmitted, long-distance wireless signals. Marconi in 1928 noted, with respect to wireless communications, that "... times of bad fading practically always coincide with the appearance of large sun-spots and intense aurora-boreali usually accompanied by magnetic storms ..." He also wrote that these were "... same periods when cables and land lines experience difficulties or are thrown out of action". Such concerns have persisted throughout the twentieth century (e.g. Gassmann, 1963 [21]), with considerable present-day research on the ionosphere being motivated by engineering considerations similar to those encountered in the early days of trans-ocean wireless communications.

The March 24, 1940 storm caused a temporary disruption of electrical service in New England, New York, Pennsylvania, Minnesota, Quebec and Ontario. A storm on February 9-10, 1958 caused a power transformer failure at the British Columbia Hydro and Power Authority. On August 2, 1972 , the Bureau of Reclamation power station in Water- 
town, South Dakota was subjected to large swings in power line voltages up to 25,000 volts. Similar voltage swings were reported by Wisconsin Power and Light, Madison Gas and Electric, and Wisconsin Public Service Corporation. A 230,000-volt transformer at the British Columbia Hydro and Power Authority exploded, and Manitoba Hydro in Canada recorded power drops from 164 to 44 megawatts in a matter of a few minutes, in the power it was supplying to Minnesota (St. Odenwald, 1998).

\section{Aurora}

The aurora is beautiful, spectacular, splendid, and appears quite frequently - almost nightly - in the polar sky [22]. Appearing in the form of majestic, colourful, irregular lights in the night sky, the aurora has a variety of shapes, colours, and structures (alike shimmering, colourful curtains), and continuously changing in the time (see Figure 8). Although many theories existed, it wasn't until a hundred years ago that scientists discovered that they were caused by interactions with the Sun. It is a large-scale electrical discharge phenomenon in the high-altitude atmosphere, resulting from quantum leaps in oxygen and nitrogen atoms.

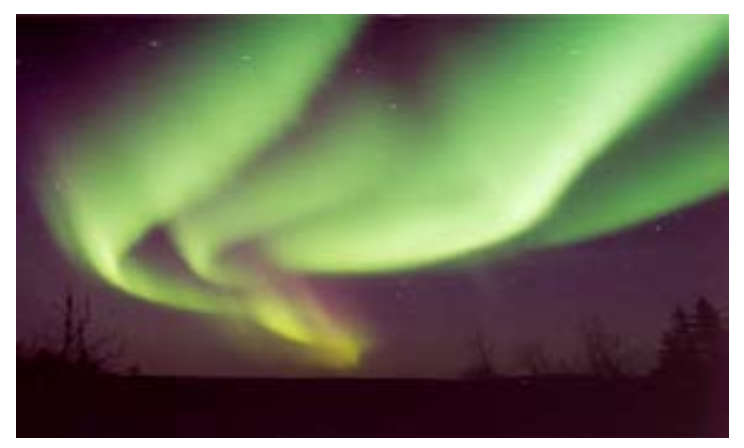

Fig 8. Auroral bands. Aurora appear from Earth as shimmering, dancing lights in the night sky. Only 100 years ago did scientists discover that the Sun was the source of these mysterious lights. Although green is the most common color, red and yellow hues are also observed. The most powerful displays occur when large clouds of particles from CMEs slam into our magnetosphere, but the constant outpouring of solar particles (called the solar wind) can cause them as well (photograph by Jan Curtis; http://soho.esac.esa.int/spaceweather/lenticular; http://www.geo.mtu.edu/ weather/aurora/images/aurora/jan.curtis/).

What exactly happens? In the highest reaches of the atmosphere, above about a hundred kilometres, oxygen and nitrogen atoms and molecules are energized and/or ionised by energetic electrons. In this transition region between the earth's atmosphere and near-earth space free electrons abound. Accelerated by electric fields in the magnetosphere, energetic electrons streaming geomagnetic field lines hit and excite atoms and molecules. The auroral light results from the de-excitation of these particles. The colour, shape, and intensity depend on the electromagnetic forces that shoot electrons downward into the upper atmosphere.

\section{Impacts of Solar-Terrestrial Processes on Technology}

During a space weather storm electric currents flowing in the magnetosphere and ionosphere change rapidly. The variations produce temporal changes in the geomagnetic field. These changes are known as (geo)magnetic disturbances or storms. According to Faraday's law of induction, magnetic disturbances are accompanied by an electric field, which drives currents within the conducting earth. These currents affect the magnetic disturbance and the (geo)electric field occurring at the earth's surface, too. The electric field also creates currents in man-made conductor systems, such as electric power transmission networks, oil and gas pipelines, telecommunication cables and railway equipment, in which they are called geomagnetically induced currents (GIC). Inconveniences to the system may result from GIC [23]. Large GIC occur most frequently in the auroral regions, in particular in North America. The increasing number of technological systems vulnerable by GIC approaching sunspot maximum with a higher geomagnetic activity make GIC research very actual and important. In Table 1, many of technological systems are listed, which must include processes and parameters from the solar-terrestrial environment in their design and/or operations. These systems are grouped into categories that have similar physical origins. Some of the effects of the solar-terrestrial environment on technical systems deployed on the Earth's surface and in space, and/or whose signals propagate through the space environment are depicted in the paper of L. Lanzerotti et al. (1999) [19], and illustrated in Figure 9 ([7], [8], [9], [10]).

Table 1 Impacts of Solar-Terrestrial Processes on
Technology
- Ionosphere Variations
" Wireless signal reflection, propagation, attenuation
" Communication satellite signal interference, scintillation
" Interference with geophysical prospecting
" Source of electrical currents in the Earth Power distribu
tion systems
Long communications cables, land and ocean Pipelines
- Radiation
" Solar cell damage
" Semiconductor device damage and failure
- Misoperation of semiconductor devices
- Spacecraft charging, surface and interior materials
- Astronaut safety
- Airline passenger safety
- Magnetic field variations
- Solar radio bursts
" Attitude control of spacecraft
" Compasses

Electric Power Systems: As compared to the 50 (or 60) $\mathrm{Hz}$ frequency used in electric power transmission, geomagnetic variations are slow with typical frequencies in the $\mathrm{mHz}$ range. Therefore GIC, when flowing through a transformer, affects as a dc current. In normal conditions the ac exciting current needed to provide the magnetic flux for the voltage transformation in a power transformer is only a few amperes, and the transformer operates within the range where the dependence of the exciting current on the voltage is linear. However, the presence of GIC implies an offset of the operation curve resulting in saturation of the transformer during one half of the ac cycle and in an extremely large non-linear exciting current (even some hundreds of amperes). The exciting current is asymmetric with respect to 
the ac half-cycles and is thus distorted by even and odd harmonics, which in turn may cause relaying problems in the system. The increased exciting current also produces large reactive power losses in the transformer contributing to a serious voltage drop. The harmonics and the reactive power demands also affect the transformer itself. The noise level is increased, and due to the saturation of the core, the magnetic flux goes through other parts of the transformer possibly resulting in overheading. The hot spots may permanently damage the insulators and cause gassing of transformer oil resulting in serious internal failures (more information in the paper of R. Pirjola et al. [23].

Perhaps the most dramatic and famous GIC failure occurred in the Hydro-Quebec power system on March 13, 1989 [24] so that for nine hours, large portions of Quebec were plunged into darkness.

Oil and Natural Gas Pipelines: GIC flowing in pipelines are known to enhance the rate of corrosion over time, and this can have catastrophic effects [25].

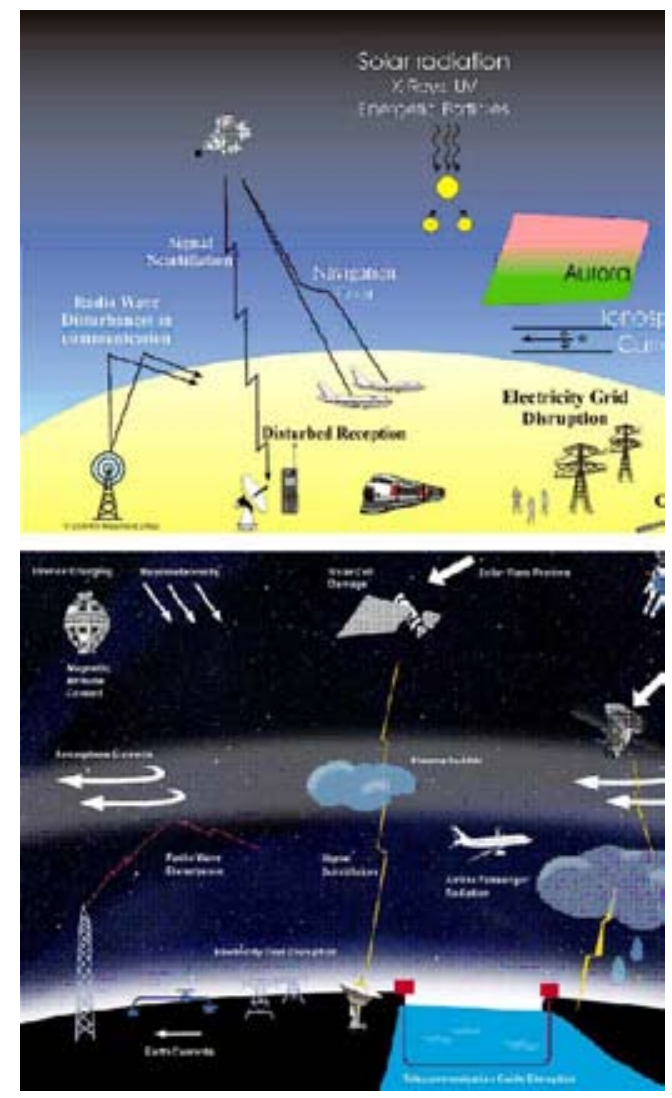

Fig 9. Some of the effects of the solar-terrestrial environment on technical systems deployed (see [7], [8], [9], [10], [19])

Effects on Spacecraft and Aircraft Electronics: Spacecraft systems are vulnerable to space weather through its influence on energetic charged particle and plasma populations, while aircraft electronics and aircrew are vulnerable to cosmic rays and solar particle events. These particles produce a variety of effects including total dose, lattice displacement damage, single event effects (SEE), noise in sensors and spacecraft charging [26]. Dose is used to quantify the effects of charge liberation by ionisation and is defined as the energy deposited as ionisation and excitation per unit mass of material. [SI units: $\mathrm{J} / \mathrm{kg}$ or grays $(=100 \mathrm{rads}$, where $1 \mathrm{rad}=100 \mathrm{erg} / \mathrm{g}$ )]. The majority of effects depend on rate of delivery and so dose-rate information is required. Accumu- lated dose leads to threshold voltage shifts in CMOS due to trapped holes in the oxide and the formation of interface states. In addition increased leakage currents and gain degradation in bipolar devices can occur. A proportion of the energy-loss of energetic radiation gives rise to lattice displacement damage and it is found that effects scale with NIEL (non-ionising energy loss per unit). Examples of damage effects are reduction in bipolar transistor gain, reduced efficiencies in solar cells, light emitting diodes and photodetectors, charge transfer inefficiency in charge coupled devices and resolution degradation in solid-state detectors. The primary cosmic rays are very energetic and are highly ionising, which means that they strip electrons from atoms, which lie in their path and hence generate charge. The density of charge deposition is proportional to the square of the atomic number of the cosmic ray so that the heavier species can deposit enough charge in a small volume of silicon to change the state of a memory cell, a "one" becoming a "zero" and vice versa. Thus memories can become corrupted and this could lead to erroneous commands. Such errors are referred to as single event upsets (SEU). Sometimes a single particle can upset more than one bit to give what are called multiple bit upsets (MBU). Certain devices could be triggered into a state of high current drain, leading to burn-out and hardware failure; such effects are termed single event latch-up or single event burn-out. These deleterious interactions of individual particles are referred to as single event effects (SEE). A classic example of hardware failure occurred in the PRARE instrument carried on the ERS-1 (European Ranging Sensing Spacecraft). Surface electrostatic charging can occur when spacecraft are bathed in energetic plasmas (several $\mathrm{keV}$ electron temperature) without the presence of neutralising cold plasma. Numerous anomalies have occurred from both surface and deep dielectric charging. Some of these have proved fatal (e.g. ANIK E1). Spurious counts, which constitute the background noise, are produced in many detector systems and these depend on the size distribution of individual depositions and can occur from both prompt ionisation and delayed depositions due to induced radioactivity. In the last ten years it has been realised that single event effects will also be experienced by sensitive electronics (avionics) in aircraft systems, which are subjected to increasing levels of cosmic radiation and their secondaries as altitude increases.

\section{Radiobiology in Space Research}

For high-altitude flights beyond the magnetic shielding of the earth, cosmic radiation i.e. high-energy particles from protons to iron ions predominate. Space radiation reaches its maximum values at a solar particle event where the lethal doses can be delivered within an hour or less [27]. These events however are rather unlikely. Much more important is the permanent i.e. protracted exposure to the low-dose radiation of the heavy charged particles of cosmic galactic rays. Because of their high local dose these particles are able to create local damage in bio-molecules that can manifest itself in long-term alterations like genetic mutation and cancer induction. It is the induction of these biological changes that determines the general risk of long-term missions. For low altitude flights such as MIR or space station orbits, trapped electrons and protons from solar origin predominate. To study the biological radiation response, especially genetic alterations and cancerogenesis, X-ray experiments can be performed in order to mimic sparsely ionising electrons. The 
radiation environment is present both on earth and in space but differs in quality and intensity. Spaceflights are on average 300 times more exposure-intensive than our daily life. Depending on dose, acute or long-term effects can be induced by radiation exposure. Acute effects like nausea, vomiting, skin irritation, depletion of white blood cells occur at doses of about 1.5 Gy or more [27]. These high doses are produced by solar storms. Long-terms effects are genetic alterations, cancer induction, damage to the central nervous system and peripheral neurons and accelerated aging. Among these effects cancerogenesis and neural damage seems to be the most important. The uncertaincies for the risk determination with regard to long-term effects are large and the risk estimation is mainly based on epidemiological data from the atomic bomb survivors.

Magnetic storms trigger myocardial infractions with mechanisms relating to heart rate variability [28].

\section{Travelling outside of the Earth's atmosphere - Astro- nauts}

Travelling outside of the Earth's atmosphere, places one in extremely hostile surroundings. Space can be defined in many ways. Using the threshold at the point where humans can no longer survive without life support, space begins in the stratosphere $(18 \mathrm{~km}$ to $50 \mathrm{~km})$. Travelling beyond the stratosphere, astronauts encounter several more layers of Earth's atmosphere before reaching the exosphere (above $300 \mathrm{~km}$ ) and the vacuum of low-Earth orbit. Heat is transferred only by radiation in space, and temperature can vary from extremes near absolute zero ( -273 degrees Celsius) to over 1200 degrees Celsius. When humans travel into space, temperature is not the only consideration. Two forms of radiation - electromagnetic and ionizing - are prevalent. Ionizing radiation, composed of high energy particles and photons, can be further categorized into the radiation found in the Van Allen Belts, solar cosmic rays (SCR), and galactic cosmic rays (GCR). All three can be potentially harmful to astronauts in space.

Van Allen Belts: The Van Allen radiation results from electrons and ions trapped in the Earth's magnetic field. They form donut shaped rings around the Earth and are distributed nonuniformly within the magnetosphere. The two belts are located at altitudes of 300 to $1200 \mathrm{~km}$ and above $10,000 \mathrm{~km}$. Extended stays in either can be fatal.

Solar Cosmic Rays: Regular and irregular forms of solar cosmic rays, or solar particle events (SPR), occur as solar wind and solar flares, respectively. This phenomenon contributes to the Van Allen Belts. Solar flares, resulting from "storms" in the Sun's magnetosphere, yield extremely high radiation doses of radiation ranging from hours to days.

During the Apollo program, there were several nearmisses between the astronauts walking on the surface of the Moon and a deadly solar storm event. The Apollo 12 astronauts walked on the Moon only a few short weeks after a major solar proton flare would have bathed the astronauts in a 100 rem blast of radiation. Another major flare that occurred half way between the Apollo 16 and Apollo 17 moonwalks would have had a much more deadly outcome had it arrived while astronauts were outside their spacecraft playing golf. Within a few minutes, the astronauts would have been killed on the spot with an incredible 7000 rem blast of radiation.

The MIR space station has been inhabited for over a decade, and according to Astronaut Shanon Lucid, the daily dosage of radiation is about equal to 8 chest X-rays per day. During one solar storm towards the end of 1989, MIR cosmonauts accumulated in a few hours, a full- years dosage limit of radiation. Meanwhile, the Space Station will be assembled in an orbit, which will take it through the South Atlantic Anomaly. Moreover, Space Station assembly will involve several thousand hours of space walks by astronauts. The main construction work will occur between the years 2000 and 2002 during the sunspot maximum period of Cycle 23. We can expect construction activity to be tied to solar conditions in a way that will frustrate the scheduling of many complex activities and the launches of Space Station components.

Galactic Cosmic Radiation Having the highest energy of the three forms, GCR consists of protons, a-particles, and heavy nuclei and is the most penetrating. It is emitted from distant stars and galaxies, diffusing through space and arriving at Earth in all directions. The flux of GCR is indirectly related to the solar cycle, with the minimum occurring at solar maximum (when the solar particles can best scatter the GCR from Earth.) Extra-vehicular activity in low-Earth orbit is shielded from this form of radiation, but in-transit crewmembers to the Moon or Mars would be susceptible to its effects.

\section{Impacts on Earth's Climate}

Scientists increasingly suspect that solar activity affects more than just satellites and power grids. Although sunspots and active regions themselves produce only minor variations in the energy output from the Sun, the magnetic activity that accompanies these regions can produce dramatic changes in the ultraviolet and soft X-ray emission levels. These changes over the solar cycle have important consequences for the Earth's upper atmosphere and are known to alter the dynamics, temperature and chemistry (e.g., ozone) in these layers. This may have implications on the Earth's climate [18]. Moreover, there have been suggestions that climate is related to the appearance and disappearance of sunspots. Researchers have found that the solar constant $\left(\mathrm{s}=1.37 \mathrm{~kW} / \mathrm{m}^{2}\right)$, which describes the solar radiation that falls on an area above the atmosphere at a vertical angle, doesn't remain constant, but varies slightly with sunspots and other solar activity. The total solar invariance varies just as regularly as the sunspot activity over the 11-year-solar cycle. Satellite measurements showed that the solar output variation is proportional to sunspot numbers. A research of Lane at al. [29] indicates that the combined effects of sunspot-induced changes in solar irradiance and increases in atmospheric greenhouse gases offer the best explanation yet for the observed rise in average global temperature over the last century. Using a global climate model based on energy conservation, Lane et al. constructed a profile of atmospheric climate "forcing" due to combined changes in solar irradiance and emissions of greenhouse gases between 1880 and 1993. They found that the temperature variations predicted by their model accounted for up to $92 \%$ of the temperature changes actually observed over the period -- an excellent match for that period. Their results also suggest that the sensitivity of climate to the effects of solar irradiance is about $27 \%$ higher than its sensitivity to forcing by greenhouse gases. The long term increase in the Sun's level of activity (both variations in the emitted energy and its magnetic fields) may have played a significant role in the measured global warming the last 150 years. It is important to quantify this effect before one 
can determine any human influences on our climate. Thus, it is of great importance to understand how the Sun works and how it varies over time so that we can better understand how it will affect us in the future.
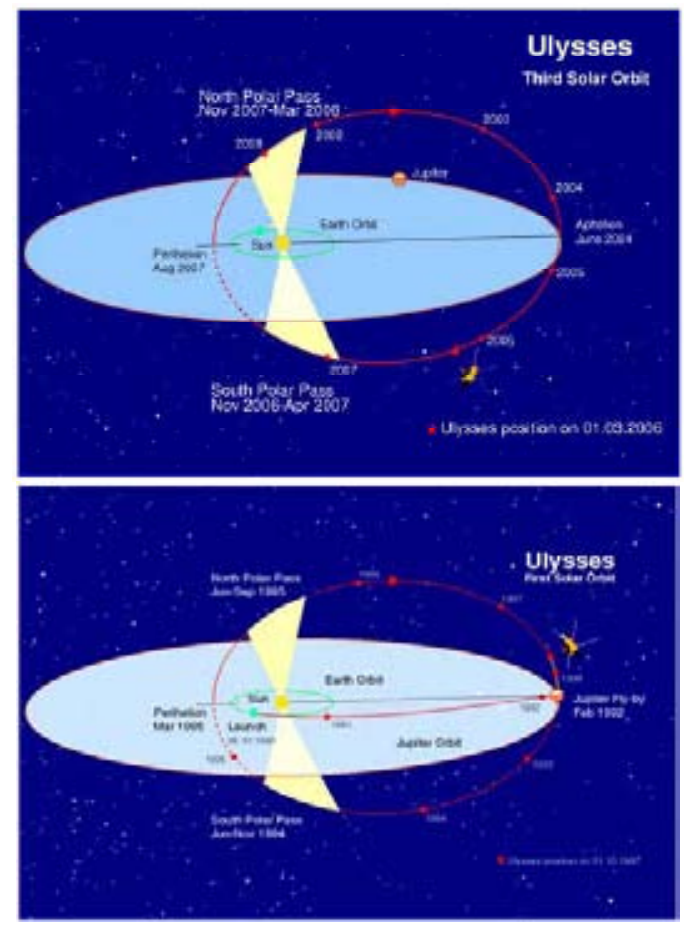

Fig 10. ULYSSES mission. First and \&third solar orbit. (http://helio.estec.esa.nl/ulysses/archive/)

\section{Perspectives in the future: Space Weather Forecast}

Recently there has been a revolution in understanding the Sun due to two major advances. The first is in theoretical modelling of the way the Sun's magnetic field interacts with solar matter. The second advance is a series of observational discoveries from three space born solar satellites: ULYSSES, Yohkoh (a Japan-USA-UK Satellite), SOHO (the Solar and Heliospheric Observatory, a joint ESA-NASA project), and TRACE (NASA). These missions are providing high resolution observations of the Sun using sophisticated imaging telescopes and spectrographs that separates out observed light into the colours it is made of. This coordinated attack on solar physics has provided breathtaking new views of the Sun and a wealth of information.

The ULYSSES Mission (Figure 10) is a joint undertaking between the European Space Agency (ESA) and the National Aeronautics and Space Administration (NASA). Its goal is the exploration of the Sun's environment far out of the ecliptic plane. ULYSSES is the only spacecraft to have visited this unique region above and below the poles of the Sun.

SOHO has been leading the way into a new era in the field of helioseismology - a study of the solar interior through the analysis of vibrations on the surface.

The fact that the Sun is affecting us in so many ways makes it very important to learn more about our own star. We need to monitor it continuously to better understand the solar cycle and any long term changes in the Sun's activity level.

\section{Conclusion}

The space is characterized by the interaction of energetic charged particles with electric and magnetic fields. In the vicinity of Earth, most of these charged particles derive their energy ultimately from the sun or from the interaction of the solar "wind" with the earth's magnetosphere. These interactions are complex, because the magnetic and electric fields that determine the motion of the particles are affected in turn by the motion of these particles. As the human exploration continues to expand space, understanding effects of our active Sun and "space weather" on power and communications, astronaut safety, satellite operations, and climate change, become day after day more important, and justifies successfully the claim "the space constitutes a natural laboratory of electrotechnics".

\section{References}

1. Wikipedia, the free encyclopedia, http://wikipedia.org

2. Kivelson, M.G., Russell, C.T., Introduction to Space Physics, Cambridge Univ. Press (1995).

3. Marhavilas, P.K., Elaboration and analysis of energetic particle observations by the ULYSSES spacecraft, in the vicinity of magnetohydrodynamic surfaces, Ph. D. thesis, Space Research Lab., Dpt of Electrical \& Computer Engineering, Democritus Univ. of Thrace, Xanthi, Greece (2004).

4. Graham, J.F., "Space exploration from talisman of the past to gateway fro the future", Chapter 4: The Solar System, Book, University of North Dakota, www.space.edu/projects (1995).

5. Hundhausen, A.J., Coronal Expansion and Solar Wind, SpringerVerlag Berlin Heidelberg New York (1972).

6. Vilmer, N., "Solar activity: Flares; CMEs; SEPs; Solar Wind", ESA WPPP-155 Proceedings of ESA Workshop on Space Weather, 11-13 Nov. 1998, ESTEC, Noordwijk, The Netherlands (1998).

7. NASA-ESA's NP-2002-8-501-GSFC Multimedia Presentation, "The dynamic Sun", developed by SOHO, http://sohowww.nasacom.nasa.gov (2002).

8. NASA's EP-1998-03-345-HQ brochure, "Storms from the Sun", (addr.: NASA/GSFC, Greenbelt MD 20771) (1998).
9. NASA's EW-2000-10-004-GSFC brochure, "New views of the Sun", (addr.: NASA/GSFC, Greenbelt MD 20771) (2000).

10. Jansen, F. and R. Hippler, "Space Weather", An interactive and science museum edition in CD-ROM, Edition II, Ernst-MoritzArndt-Univ. Greifswald, Institute for Physics, Germany (2003).

11. Pulkkinen, "Magnetospheric electrodynamics: Energetic particle signatures of geomagnetic storms and substorms", ESA WPPP155 Proceedings of ESA Workshop on Space Weather, 11-13 Nov. 1998, ESTEC, Noordwijk, The Netherlands (1998).

12. Space Environments \& Effects Program, NASA Marshall Space Flight Center (http://see.msfc.nasa.gov/pf/pf.htm)

13. Rostoker, G., S.-I., Akasofu, J. Foster, R.A. Greenward, Y. Kamide, K. Kawasaki, A.T.Y. Lui, R.L. McPherron, and C.T. Russel, Magnetospheric substorms-definitions and signatures, J. Geophys. Res., 85, 1663 (1980).

14. McPherron, R.L., Physical processes producing magnetospheric substorms and magnetic storms, in Geomagnetism, vol. 4, p.593, Academic Press, San Diego, CA, (1991).

15. Gonzalez, W.D., J.A. Joselyn, Y. Kamide, H.W. Kroehl, G. Rostoker, B.T. Tsurutani, and V.M. Vasyliunas, What is a geomagnetic storm?, J. Geophys. Res., 99, 5771, (1994). 
16. Baker, D.N., T.I. Pulkkinen, X. Li, S. G. Kanekal, J.B. Blake, R.S. Selesnick, M.G. Henderson, G.D. Reeves, and H. E. Spence, Coronal mass ejections, magnetic cloudes, and relativistic magnetospheric electron events: ISTP, J. Geophys. Res., 103, 17292, (1998).

17. Frank, A., "Virtual space weather from the Sun", Insights Magazine, (http://www-personal.engin.umich.edu/), (1999).

18. Brekke, P., Space Weather, Europhysics News, Vol. 32, No 6, (2001).

19. Lanzerotti, L.J., Thomson, D.J., and Maclennan, C.G., "Engineering issues in space weather", Modern Radio Science, pp. 2550, (1999).

20. Carrington, R.C., Observation of the spots on the sun from November 9, 1853, to March 24, 1860, made at redhill, William and Norgate, London and Edinburg, 167, (1863).

21. Gassmann, G. J., The effects of disturbances of solar origin on Communications, AGARD Avionics Panel, Macmillan Company, New York, (1963).

22. Daglis, I., and S.-I. Akasofu, "Aurora - The magnificent northern lights", The EGGS Newsletter \& Information Service of the E.G.S. Issue \#7, http://www.the-eggs.org/, pp. 12-18, (2004).

23. Pirjola, R., A. Viljanen, O. Amm and A. Pulkkinen, "Power and Pipelines (Ground Systems)", ESA WPPP-155 Proceedings of ESA Workshop on Space Weather, 11-13 Nov. 1998, ESTEC, Noordwijk, The Netherlands (1998)
24. Kappenman, J.G., and V.D. Albertson, Bracing for the geomagnetic storms, IEEE Spectrum, March 1990, pp. $27-33$ (1990).

25. Odenwald, St., "Solar Storms: The Silent Menace" (http://image.gsfc.nasa.gov/poetry/storm/ storms.html) (1998).

26. Dyer, C., and D. Rodgers, "Effects on Spacecraft and Aircraft Electronics", ESA WPPP-155 Proceedings of ESA Workshop on Space Weather, 11-13 Nov. 1998, ESTEC, Noordwijk, The Netherlands (1998).

27. Testard, I., L. Sabatier, S. Ritter, M. Durante and G. Kraft, "Radiobiology for Space Research", ESA WPPP-155 Proceedings of ESA Workshop on Space Weather, 11-13 Nov. 1998, ESTEC, Noordwijk, The Netherlands (1998).

28. Halberg, F., Cornelissen G., Engebretson, M., Siegelova, J., Schwartzkopff, O., "Transdisciplinary biological- heliogeophysical relations at weekly, half-yearly and schwabe- and halecycle frequencies", Proceedings of the Noninvasive Methods in Cardiology Symposium held as a part of the MEFA Congress Progress in Medicine and Pharmacy, Brno, November 3-6, 1999, Scripta Medica, (BRNO)-74(2):69-74 (2001).

29. Lane, L.J., M.H. Nichols, and H.B. Osborn, "Time series analyses of global change data", Environ. Pollut., 83, 63-68 (1994).

30. Baker, D.N., T.I. Pulkkinen, V. Angelopoulos, W. Baumjohann, and R.L. McPherron, The neutral line model of substorms: Past results and present view, J. Geophys. Res., 101, 12,975 (1996).

31. Hundhausen, A.J., in "Introduction to Space Physics", eds Kivelson, M.G., Russell, C.T., 91 (1995). 